\title{
VI. The effective resistance and inductance of a helical coil
}

\author{
J.W. Nicholson M.A. D.Sc.
}

To cite this article: J.W. Nicholson M.A. D.Sc. (1910) VI. The effective resistance and inductance of a helical coil , Philosophical Magazine Series 6, 19:109, 77-91, DOI: 10.1080/14786440108636778

To link to this article: http://dx.doi.org/10.1080/14786440108636778

册 Published online: 21 Apr 2009.

Submit your article to this journal $\sqsubset \pi$

Џ Article views: 3

Q View related articles $\square$

7 Citing articles: 7 View citing articles $\square$ 
of the rods and cones on the retina (only the main essentials of which are referred to in this paper), seem to be still the subject of much discussion among physiologists to whom we must look for the exact interpretation of their bearing on problerus in photometry. Dr. Edridge Green, for instance, in a recent lecture before the Optical Society *, expressed his dissent from the theory of vision based on the supposed behaviour of the rods and cones, and expressed the view that visual impulses are only received through the latter organs. But the general phenomena on which the theory mainly rests, and which have been utilized in this paper, seem to be well authenticated.

VI. The Effective Resistance and Inductance of a Helical Coil. By J. W. Nicholson, M.A., D.Sc. $\dagger$

$r$ HE problem of the propagation of alternating currents 1 in wires has been solved in but few cases. For a single wire isolated in space, a solution has been given by Sommerfeld $\ddagger$. If the return current be conducted along a concentric sheath, instead of by the dielectric, the problem is that treated earlier by Sir J. J. Thomson \$. The case in which the return current flows along a parallel wire has been discussed by Mie $\|$, and to a first approximation, in a simpler manner, by Morton $\uparrow$, who has given, in other papers $^{* *}$, a detailed examination of the distribution of forces, and applied his method also to the case of a greater number of wires.

The influence of electrostatic capacity in wires renders the mathematical investigations very complicated, but from a practical point of view, the main object to be attained is a knowledge of the effective inductance and resistance of the wires when the capacity and leakage are not so great as to produce a sensible attenuation of the wave in a short distance.

The concentration of the current in the outer regions of the wires, when the frequency is high, completely changes the character of the inductance and resistance, and a knowledge of their values for steady currents is frequently of

* Optical Society (London) Oct. 21, 1909.

+ Communicated by the Physical Society : read November 26, 1909.

$\ddagger$ Wied. Ann. lxvil. p. 233 (1899).

$\$$ Recent Researches, p. 262.

\|l Ann. der Phys. ii. p. 202 (1900).

- Phil. Mag. Dec. 1900.

** Phil. Mai. May 1901, Sept. 1902, June 1903. 
little service. But even with the restriction to wires with small capacity and leakage, mathematical solutions have not hitherto progressed far. The problem of the concentric main was discussed by Maxwell ${ }^{*}$ and Heaviside $\dagger$, and afterwards for very high frequencies by Lord Rayleigh $\ddagger$. A practical formula for the resistance of the inner conductor was given by Lord Kelvin \$, whose solution is not essentially different from that of Heaviside.

Formulæ for the effective inductance in this case have also been obtained by Sir J. J.Thomson II, and an exhaustive treatment of the whole problem by more simple methods, in which the ranges of application of all the previous formulx are critically examined, has been given by Russell $\uparrow$.

The investigation of the effective inductance and resistance of two parallel wires, one being the return of the other, was made by the author **, and developed, in a later paper $\dagger \dagger$, in a form capable of more immediate practical use. The upper limit necessary to the capacity was at the same time examined.

The main problem of importance in which the wires are bent is that of the single helical coil of considerable length. Solutions have hitherto dealt with the case in which the wire is closely wound round a circular cylinder, forming a helix whose pitch is very small. Wien $\ddagger \ddagger$ first discussed the problem, but assumed that the current distribution is the same at all points equidistant from the axis. Battelli showed that this assumption was not lawful, and the results do not agree with experiment. Shortly afterwards, Sommerfeld $\S \S$ investigated the problem in so far as the resistance is concerned, and his method was applied later by Coffin \|\| to a determination of inductance. Picciati $\Upsilon$ T gave an independent solution, but like those of Sommerfeld and Wien, its agreement with experiment was not very good. The best solution in this respect is that of Cohen ${ }^{* * *}$, which appears to be quite

* Elec. and Mag. vol. ii. $\$ 690$.

+ Electrical Papers, i. p. 353 ; ii. p. 64 et se\%.

I Phil. Mag. xxri. p. 381 (1886); Scientific Papers, ii. p. 486.

\$ Journ. Inst. Elec. Eng. xxiii. p. 4 (1889) ; Math. \& Phys. Papers, iii.p. 491.

1) Recent Researches, p. 205.

T) Phil. Mag. April 1909; Proc. Phys. Soc. xxi.

** Phil. MLag. Feb. 1909 ; Proc. Phys. Soc. xxi.

t十 Phil. Mag. Sept. 1909; Proc. Phys. Soc. xxii.

It Ann. der Phys. xiv. p. 1 (1904).

$\S \S$ Ann.der Phys. xv. p. 673 (1904).

ili Bulletin of Bureau of Standards, ii. p. 275 .

99 Il Nuovo Cimento (5) ii. p. 35.

*** Bulletin of Bureau of Standards, iv. no. 1 . 
satisfactory for all cases in which the pitch of winding is very small.

The present paper deals with the corresponding formulx for a helical wire whose pitch is not very small, wound on a cylinder whose radius is large compared with that of the section of the wire, which is of course circular, and cannot be treated as square in the manner applied by Cohen to the other extreme case. A rigorous solution would be very difficult, but it is shown that some very simple approximate results are sufficient for practical purposes. The coil is to be regarded as sufficiently long for the effects of the ends to be neglected. A connexion between these formula and those of Cohen cannot be made without a consideration of the difficult intermediate case in which the pitch is moderately small.

\section{Choice of Coordinates.}

Let the axis of the cylinder on which the wire is wound be chosen as that of $z$ in a Cartesian system. Defining the central curve as the locus of the centres of all normal sections of the wire, this curve will be a helix, and if $a$ is the radius of the cylinder, and $\theta$ the twist in a plane perpendicular to $z$ of the radius in that plane measured from a line parallel to $x$, the coordinates of a point on the helix become

$$
x=a \cos \theta, \quad y=a \sin \theta, \quad z=a \theta \tan \alpha . .
$$

where $\alpha$ is the angle of the helix.

Let $(\rho \phi)$ be polar coordinates in the plane of the normal section at the point defined by $\theta$. The initial line from which $\phi$ is to be measured is perpendicular to $z$ and to the tangent of the helix. Thus if $z$ were vertical, the initial line in any section would be the horizontal line. Now the direction cosines, at the point $\theta$, of the tangent to the helix are

$$
(-\sin \theta \cos \alpha, \cos \theta \cos \alpha, \quad \sin \alpha) .
$$

A line perpendicular to this and to the axis of $z(001)$ is readily shown to bave a direction

$$
(\cos \theta, \sin \theta, 0) \text {. }
$$

This is the line $\phi=0$ in the section defined by $\theta$.

Let $(\lambda, \mu, \nu)$ be the direction cosines of the radius vector $\rho$ in the section. It is at an angle $\phi$ to the above line, and is perpendicular to the central curve, so that

$$
\begin{gathered}
\lambda \cos \theta+\mu \sin \theta=\cos \phi, \\
-\lambda \sin \theta \cos \alpha+\mu \cos \theta \cos \alpha+\nu \sin \alpha=0, \\
\text { with } \quad \lambda^{2}+\mu^{2}+\nu^{2}=1 .
\end{gathered}
$$


Thus

$$
\begin{aligned}
1-\nu^{2}=\lambda^{2}+\mu^{2} & =(\lambda \cos \theta+\mu \sin \theta)^{2}+(\mu \cos \theta-\lambda \sin \theta)^{2} \\
& =\cos ^{2} \phi+\nu^{2} \tan ^{2} \alpha,
\end{aligned}
$$

or $\quad \nu= \pm \cos \alpha \sin \phi$.

We choose the positive sign, and deduce

$$
\left.\begin{array}{l}
\lambda=\cos \theta \cos \phi+\sin \alpha \sin \theta \sin \phi, \\
\mu=\sin \theta \cos \phi-\sin \alpha \cos \theta \sin \phi, \\
\nu=\cos \alpha \sin \phi
\end{array}\right\} .
$$

as the direction of the radius vector.

The coordinates of any point of space are therefore

$$
\begin{aligned}
x & =a \cos \theta+\rho \lambda \\
& =(a+\rho \cos \phi) \cos \theta+\rho \sin \alpha \sin \theta \sin \phi, \\
y & =a \sin \theta+\rho \mu \\
& =(a+\rho \cos \phi) \sin \theta-\rho \sin \alpha \cos \theta \sin \phi, \\
z & =a \theta \tan \alpha+\rho \nu \\
& =a \theta \tan \alpha+\rho \cos \alpha \sin \phi
\end{aligned}
$$

We shall call $(\rho \phi \theta)$ the helical coordinates of any point of space. If $\delta s$ (which only involves $\delta \theta$ ) be an element of length perpendicular to a normal section at the point $(\rho, \phi, \theta)$, then the space elements $\delta s, \delta \rho, \rho \delta \phi$ are mutually perpendicular, and the surfaces $\rho=$ const. are those of helical wires with a common central curve. This system of coordinates is therefore appropriate to problems in which surface conditions must be satisfied at the boundaries of such wires.

\section{Property of Orthogonal Systems.}

In the problem here contemplated, in which an alternating electromotive force $\mathrm{E} e^{p t}$ is applied to the terminals of a long helical coil, the electric and magnetic vectors in and near the coil will not depend on $\theta$, if the other portion of the circuit is kept at a distance. Thus $\partial / \partial \theta=0$.

Consider now a general orthogonal system in which the components of all vectors are independent of one coordinate. Denoting the three coordinates by $(\rho \phi \theta)$, let the corresponding space elements along their instantaneous directions of increase at any point be

$$
\left(d s_{1}, d s_{2}, d s_{3}\right)=\left(d \rho / p_{1}, d \phi / p_{2}, d \theta / p_{3}\right) . . .
$$

Let $(\alpha, \beta, \gamma),(x, y, z)$ be the components of magnetic and 
electric force along these directions. Since the operation $\partial / \partial \theta$ annuls any component, the solenoidal conditions become.

$$
\left.\begin{array}{l}
\frac{\partial}{\partial \rho} \frac{\mathrm{X}}{p_{2} p_{3}}+\frac{\partial}{\partial \phi} \frac{\mathrm{Y}}{p_{1} p_{3}}=0, \\
\frac{\partial \alpha}{\partial \rho p_{2} p_{3}}+\frac{\partial}{\partial \phi} \frac{\beta}{p_{1} p_{3}}=0 .
\end{array}\right\} \quad \cdot . \cdot
$$

It is assumed also that $\left(p_{1} p_{2} p_{3}\right)$ are independent of $\theta$, as is the case in all important applications. Functions $\chi$ and $\psi$ may be introduced, where

$$
\left.\begin{array}{cc}
\mathrm{X}=p_{2} p_{3} \partial \chi / \partial \phi, & \mathrm{Y}=p_{1} p_{3} \partial \chi / \partial \rho \\
\alpha=p_{2} p_{3} \partial \psi / \partial \phi, & \beta=p_{1} p_{3} \partial \psi / \partial \rho
\end{array}\right\} .
$$

By Faraday's law, if $\mu$ be the permeability,

$$
\begin{aligned}
\frac{-\mu}{p_{1} p_{3}} \partial t & =\frac{\partial}{\partial \rho} \frac{\mathrm{Y}}{p_{2}}-\frac{\partial}{\partial \phi} \frac{\mathrm{X}}{p_{1}} \\
& =\frac{\partial}{\partial \rho} \frac{p_{1} p_{2}}{p_{3}} \frac{\partial \chi}{\partial \rho}+\frac{\partial}{\partial \phi} \frac{p_{2} p_{3}}{p_{1}} \frac{\partial \chi}{\partial \phi} ; . .
\end{aligned}
$$

and by Ampères law, in a dielectric,

$$
\frac{1}{\mathrm{~V}^{2} p_{2} p_{3}} \frac{\partial \mathrm{X}}{\partial t}=\mu \frac{\partial}{\partial \phi} \frac{\mathrm{y}}{p_{3}} ; \quad . \quad . \quad .
$$

whence, on reduction, $\chi$, andisimilarly $\psi$, satisfy the equation

$$
\frac{\partial}{\partial \rho} \frac{p_{1} p_{3}}{p_{2}} \frac{\partial \psi}{\partial \rho}+\frac{\partial}{\partial \phi} \frac{p_{2} p_{3}}{p_{1}} \frac{\partial \psi}{\partial \phi}=\frac{p_{3}}{\overline{\mathrm{V}}^{2} p_{1} p_{2}} \frac{\partial^{2} \psi}{\partial t^{2}}
$$

where $V$ is the velocity of propagation of electromagnetic disturbances. Thus any disturbance in which the vectors are independent of one coordinate of an orthogonal system must be made up of two types, in which the electric and magnetic forces along the direction of increase of that coordinate are respectively zero everywhere.

If the wave-length of the oscillation, supposed simply periodic, be $2 \pi / k$, the equation for $\psi$ becomes

$$
\frac{\partial}{\partial \rho} \frac{p_{1} p_{3}}{p_{2}} \frac{\partial \psi}{\partial \rho}+\frac{\partial}{\partial \phi} \frac{p_{2} p_{3}}{p_{1}} \frac{\partial \psi}{\partial \phi}+\frac{k^{2} p_{3}}{p_{1} p_{2}} \psi=0
$$

and for a conducting medium, it is only recessiry to change $i^{2}$. In the present problem the oscillation defined by $\psi$ exists, Phil. Mag. S. 6. Vol. 19. No. 109. Jan. 1909. 
but not that defined by $\chi$. The vectors are given by

$$
\begin{array}{lc}
\mathrm{X}=\mathrm{Y}=0, & \alpha=\gamma=0, \\
\mathrm{Z}=-\frac{p_{3} \partial \psi}{\mathrm{V}^{2}} \frac{\partial}{\partial t}, & \beta=-p_{1} p_{3} \frac{\partial \psi}{\partial \rho} .
\end{array}
$$

The electric force is along $\theta$ increasing, or parallel at any point to the corresponding tangent of the central curve. The magnetic force is along $\phi$ increasing. At the surface of any wire of the helical system, both forces are tangential, as they should be.

Solution as a Fourier Series.

If $d s_{1}$ is an element of arc along $\rho$ increasing,

$$
\left(\frac{\partial s_{1}}{\partial \rho}\right)^{2}=\left(\frac{\partial w}{\partial \rho}\right)^{2}+\left(\frac{\partial y}{\partial \rho}\right)^{2}+\left(\frac{\partial z}{\partial \rho}\right)^{2}=\lambda^{2}+\mu^{2}+\nu^{2}=1 ;
$$

so that $p_{1}=1$. Moreover,

$$
\begin{aligned}
\left(\frac{\partial s_{2}}{\partial \phi}\right)^{2} & =\left(\frac{\partial x}{\partial \phi}\right)^{2}+\left(\frac{\partial y}{\partial \phi}\right)^{2}+\left(\frac{\partial z}{\partial \phi}\right)^{2} \\
& =(-\rho \sin \phi \cos \theta+\rho \sin \alpha \sin \theta \cos \phi)^{2} \\
& +(\rho \sin \phi \sin \theta+\rho \sin \alpha \cos \theta \cos \phi)^{2}+\rho^{2} \cos ^{2} \alpha \cos ^{2} \phi=\rho^{2}
\end{aligned}
$$

on reduction, and $p_{2}=\rho^{-1}$. Similarly

$p_{3}=\left\{a^{2} \sec ^{2} \alpha+2 a \rho \cos \phi+\rho^{2}\left(\cos ^{2} \phi+\sin ^{2} \alpha \sin ^{2} \phi\right)\right\}^{-\frac{2}{2}}$.

If the wire be thin, $p_{3}=a^{-1} \cos \alpha$, and we are led to the usual solution for the straight wire of great length.

The differential equation may be reduced to

$$
\frac{\partial}{\partial \rho} \rho \frac{\partial \psi}{\partial \rho}+\frac{1}{\rho} \frac{\partial^{2} \psi}{\partial \phi^{2}}+k^{2} \rho \psi+\rho \frac{\partial \psi}{\partial \rho} \frac{1}{p_{3}} \frac{\partial p_{3}}{\partial \rho}+\frac{1 \partial \psi}{\rho} \frac{\partial}{\partial \phi} \frac{\partial p_{3}}{\partial \phi}=0 .
$$

Now

$$
\begin{aligned}
\frac{1}{p_{3}} \frac{\partial p_{:}}{\partial \phi} & =\frac{a \rho \sin \phi+\rho^{2} \sin \phi \cos \phi \cos ^{2} \alpha}{a^{2} \sec ^{2} \alpha+2 \alpha \rho \cos \phi+\rho^{2}\left(\cos ^{2} \phi+\sin ^{2} \alpha \sin ^{2} \phi\right)} \\
& =\frac{\rho}{a} \sin \phi \cos ^{2} \alpha-\frac{\rho^{2}}{2 \alpha^{2}} \sin 2 \phi \cos ^{4} \alpha+\ldots \ldots \ldots . .
\end{aligned}
$$

on expansion in powers of $\rho / a$, replacing the trigonometric functions by those of multiple angles. Again,

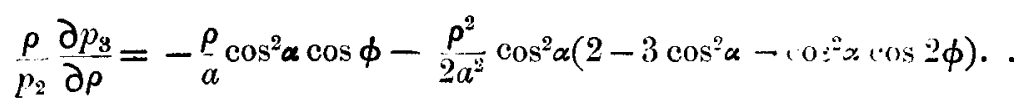

We shall not retain $\rho^{3} / a^{3}$ or higher powers. 
The equation (13) has a solution of the form

$$
\psi=A_{0}+A_{1} \cos \phi+A_{2} \cos 2 \phi+\ldots \ldots . . .
$$

where the functions $A$ are independent of $\phi$. By substitution, the accent denoting $\partial / \partial \rho$,

$\left(\frac{\partial}{\partial \rho} \rho \frac{\partial}{\partial \rho}+k^{2} \rho\right)\left(A_{0}+A_{1} \cos \phi+A_{2} \cos 2 \phi+\ldots\right)-\frac{1}{\rho}\left(A_{1} \cos \phi+2^{2} A_{2} \cos 2 \phi \ldots \ldots\right)$

$-\frac{\rho}{2 a} \cos ^{2} \alpha\left\{2 \cos \phi \mathrm{A}_{0}{ }^{\prime}+(1+\cos 2 \phi) \mathrm{A}_{1}{ }^{\prime}+(\cos \phi+\cos 3 \phi) \mathrm{A}_{2}{ }^{\prime}+\ldots \ldots\right\}$

$-\frac{\rho^{2}}{2 \alpha^{2}} \cos ^{2} \alpha\left(2-3 \cos ^{2} \alpha\right)\left(A_{0}{ }^{\prime}+\cos \phi A_{1}{ }^{\prime}+\cos 2 \phi A_{2}{ }^{\prime}+\ldots \ldots\right)$

$+\frac{\rho^{2}}{4 a^{2}} \cos ^{4} \alpha\left\{2 \cos 2 \phi \mathrm{A}_{0}{ }^{\prime}+(\cos \phi+\cos 3 \phi) \mathrm{A}_{1}{ }^{\prime}+(1+\cos 4 \phi) \mathrm{A}_{2}{ }^{\prime}+\ldots \ldots\right\}$

$-\frac{\cos ^{2} \alpha}{2 a}\left\{(1-\cos 2 \phi) A_{1}+2(\cos \phi-\cos 3 \phi) A_{2}+3(\cos 2 \phi-\cos 4 \phi) A_{3}+\ldots \ldots\right\}$

$+\frac{\rho}{4 a^{2}} \cos ^{4} \alpha\left\{(\cos \phi-\cos 3 \phi) A_{1}+2(1-\cos 4 \phi) A_{2}+3(\cos \phi-\cos 5 \phi) A_{3}+\ldots \ldots\right\}$,

we deduce the following system of equations, where

$$
\begin{array}{r}
\mathrm{D}=\frac{d}{d \rho} \rho \frac{d}{d \rho}+k^{2} \rho, \ldots . . \\
\mathrm{DA}_{0}-\frac{\rho}{2 a} \cos ^{2} \alpha \mathrm{A}_{1}{ }^{\prime}-\frac{\rho^{2}}{2 a^{2}} \cos ^{2} \alpha\left(2-3 \cos ^{2} \alpha\right) \mathrm{A}_{0}{ }^{\prime}+\frac{\rho^{2}}{4 a^{2}} \cos ^{4} \alpha \mathrm{A}_{2}{ }^{\prime} \\
-\frac{\mathrm{A}_{1}}{2 a} \cos ^{2} \alpha+\frac{\rho \mathrm{A}_{2}}{2 a^{2}} \cos ^{4} \alpha=0, \\
\mathrm{DA} \mathrm{A}_{1}-\frac{\mathrm{A}_{1}}{\rho}-\frac{\rho}{2 a} \cos ^{2} \alpha\left(2 \mathrm{~A}_{0}{ }^{\prime}+\mathrm{A}_{2}{ }^{\prime}\right)-\frac{\rho^{2}}{2 a^{2}} \cos ^{2} \alpha\left(2-3 \cos ^{2} \alpha\right) \mathrm{A}_{1}{ }^{\prime} \\
+\frac{\rho^{2}}{4 a^{2}} \cos ^{4} \alpha\left(\mathrm{A}_{1}{ }^{\prime}+\mathrm{A}_{3}{ }^{\prime}\right)-\frac{\mathrm{A}_{2}}{a} \cos ^{2} \alpha+\frac{\rho}{4 a^{2}} \cos ^{4} \alpha\left(\mathrm{A}_{1}+3 \mathrm{~A}^{3}\right)=0, .
\end{array}
$$

and so on. But it is evident that $A_{0}, A_{1}, A_{2} \ldots \ldots$ form a sequence, each member of which is of a higher order in $a^{-1}$ than those before

For the purpose of this investigation, $A_{0}$ is required to order $\rho^{2} / a^{2}$, and therefore, as an intermediary, $A_{1}$ to order $\rho / a$ only, and $A_{2}$ not at all. Therefore the equations may be written more simply in the forms

$$
\left.\begin{array}{r}
\mathrm{DA}_{0}-\frac{\rho^{2}}{2 a^{2}} \cos ^{2} \alpha\left(2-3 \cos ^{2} \alpha\right) \frac{d \mathrm{~A}_{0}}{d \rho}=\frac{\cos ^{2} \alpha d}{2 a d \rho}\left(\rho \mathrm{A}_{1}\right), \\
\mathrm{DA}_{1}-\frac{\mathrm{A}_{1}}{\rho}=\frac{\cos ^{2} \alpha}{a} \rho \frac{d \mathrm{~A}_{0},}{d \rho} \cdot . . .
\end{array}\right\}
$$

and $A_{1}$ must be eliminated. 
It is convenient to take a new variable $k \rho=x$, so that

$$
\begin{aligned}
& \left(\frac{d^{2}}{d x^{2}}+\frac{1}{x} \frac{d}{d x}+1-\frac{1}{x^{2}}\right) \mathrm{A}_{1}=\frac{\cos ^{2} \alpha}{k a} \frac{d \mathrm{~A}_{0}}{d x}, . . . . \\
& \left(\frac{d^{2}}{d x^{2}}+\frac{1}{x} \frac{d}{d x}+1-\frac{\cos ^{2} \alpha\left(2-3 \cos ^{2} \alpha\right)}{2 k^{2} a^{2}} x \frac{d}{d x}\right) \mathrm{A}_{0}=\frac{\cos ^{2} \alpha}{2 k a x} \frac{d}{d x}\left(x \mathrm{~A}_{1}\right) \ldots
\end{aligned}
$$

Now (20) may be written

or

$$
\mathrm{A}_{1}+\frac{d}{d x} \frac{1}{x} \frac{d}{d x} x \mathrm{~A}_{1}=\frac{\cos ^{2} \alpha}{k a} \frac{d \mathrm{~A}_{0}}{d x}
$$

$$
\left(\frac{1}{x} \frac{d}{d x} x \frac{d}{d x}+1\right) \frac{1}{x} \frac{d}{d x} x \mathrm{~A}_{2}=\frac{\cos ^{2} \alpha}{k a x} \frac{d}{d x} x \frac{d \mathrm{~A}_{0}}{d x},
$$

and the elimination with (21) may be effected at once, yielding

$$
\begin{aligned}
\left(\frac{d^{2}}{d x^{2}}+\frac{1}{x} \frac{d}{d x}+1\right)\left\{\frac{d^{2}}{d x^{2}}+\frac{1}{x} \frac{d}{d x}+1-\right. & \left.\frac{\cos ^{2} \alpha\left(2-3 \cos ^{2} \alpha\right)}{2 k^{2} a^{2}} x \frac{d}{d x}\right\} A_{0} \\
& =\frac{\cos ^{4} \alpha}{2 k^{2} a^{2}} \frac{1}{x} \frac{d}{d x} x \frac{d A_{0}}{d x} ; .
\end{aligned}
$$

and it is at once evident that $A_{0}$ is only altered to the second order by the bending of the wire. It is henceforth supposed that not only $a$, but $k a$ is large, as this case includes nearly all of practical interest. When $k a$ is small, a special investigation is necessary.

Ignoring the effect of bending, the differential equation becomes

$$
\left(\frac{d^{2}}{d x^{2}}+\frac{1}{x} \frac{d}{d x}+1\right)^{2} \mathrm{~A}_{0}=0
$$

so that, for a solution finite on the central curve,

and finally

$$
\left(\frac{d^{2}}{d x^{2}}+\frac{1}{x} \frac{d}{d^{\prime} x}+1\right) A_{0} \propto \mathrm{J}_{0}(x)
$$

$$
\mathrm{A}_{0}=\mathrm{AJ}_{0}(x)+\mathrm{B} x \mathrm{~J}_{1}(x)
$$

where $A$ and $B$ are arbitrary. In determining the selfinduction and resistance, we only require the modification, inside the metal, of the form $\mathrm{J}_{0}(x)$, and in the outer medium, of the form $\mathrm{K}_{0}(\iota x)$. The solution $x \mathrm{~J}_{1}(x)$ may be discarded, as it corresponds to nothing in the case of the straight wire, to which the formulæe must lead when $a$ is infinite, or $\alpha=0$. 
Writing therefore

$$
\mathrm{A}_{0}=\mathrm{J}_{0}(x)+\frac{\cos ^{2} a}{2 k^{2} a^{2}} u, . . \quad . \quad .
$$

where $u$ is a new variable, only required in its first term, not involving $(k a)^{-2}$, then on substitution, and rejection of $(k a)^{-2}$ in the result,

$$
\left(\frac{d^{2}}{d x^{2}}+\frac{1}{x} \frac{d}{d x}+1\right)^{2} u=\frac{\cos ^{2} \alpha}{x} \frac{d}{d x} x \frac{d \mathrm{~J}_{0}}{d x}+\left(2-3 \cos ^{2} \alpha\right)\left(\frac{d^{2}}{d x^{2}}+\frac{1}{x} \frac{d}{d x}+1\right) \cdot v \frac{d \mathrm{~J}_{0}}{d x} \text {. }
$$

By properties of the Bessel function of zero order, this becomes

$$
\left(\frac{d^{2}}{d x^{2}}+\frac{1}{x} \frac{d}{d x}+1\right)^{2} u=+\left(1-5 \sin ^{2} \alpha\right) \mathrm{J}_{0}(x) ; \quad .
$$

whence

$$
\left(\frac{d^{2}}{d x^{2}}+\frac{1}{x} \frac{d}{d x}+1\right) u=+\frac{1}{2}\left(1-5 \sin ^{2} \alpha\right) x \mathrm{~J}_{1}(x),
$$

ignoring the complementary function. Finally,

$$
u=-\frac{1}{8}\left(1-5 \sin ^{2} \alpha\right) x^{2} J_{0}(x), . \quad \text {. . . }
$$

again ignoring a complementary function. The total form thus ignored is $\mathrm{A} x \mathrm{~J}_{1}(x)+\mathrm{BJ}_{0}(x)$, of which the first term pertains to the redundant solution of the original equation. The second has no relation to the original $\mathrm{J}_{0}(x)$ with unity as coefficient. We write therefore

$$
\mathrm{A}_{0}=\left\{1-\frac{\cos ^{2} \alpha\left(1-5 \sin ^{2} \alpha\right) x^{2}}{16 k^{2} \alpha^{2}}\right\} J_{0}(x) .
$$

as the value of $\mathrm{A}_{0}$ to the second order. Substitution in the differential equation directly verifies this solution, and it therefore corresponds to the function $\mathrm{J}_{0}(x)$ of the unbent wire.

\section{Solution for the Dielectric.}

The above values hold for the interior of the wire of resistivity $\sigma$, provided that $k^{2}=-4 \pi \mu \nu / \sigma$, where $p / 2 \pi$ is the frequency of oscillation. For the dielectric, the appropriate function outside a straight wire would be $K_{0}(\iota h \rho)$, where $h=p / V$ takes the place of $k$. The function is defined by

$$
\mathrm{K}_{n}(\iota u)=\int_{0}^{\infty} d \phi \cosh n \phi e^{-\imath u \cosh \phi} . .
$$


and possesses the properties

$$
\left.\begin{array}{rl}
\frac{d}{d u} u n \mathrm{~K}_{n}(u) & =-u^{n} \mathrm{~K}_{n-1}(u), \\
\mathrm{K}_{n+1}(u)-\mathrm{K}_{n-1}(u) & =\frac{2 n}{u} \mathrm{~K}_{n}(u), \\
\mathrm{K}_{n+1}(u)+\mathrm{K}_{n-1}(u) & =-2 \frac{d \mathrm{~K}_{n}(u)}{d u} \\
\frac{d}{d u}-\mathrm{K}_{0}(u) & =-\mathrm{K}_{1}(u) .
\end{array}\right\} \cdot \cdot
$$

The analysis required in determining the corrected function is very similar to that above, and leads finally to the function

$$
\mathrm{B}_{0}(\iota h \rho)=\left\{1-\frac{\cos ^{2} \alpha\left(1-5 \sin ^{2} \alpha\right)}{16 \iota^{2} a^{2}} h^{2} \rho^{2}\right\} \mathrm{K}_{0}(\iota h \rho) .
$$

Determination of Inductance and Resistance.

The terms of the Fourier series involving $\phi$ do not contribute to the total current across a section of the wire. Let E $e^{\text {spt }}$ be the mean value across a section, of the line integral of impressed electric force per unit length perpendicular to the section, and parallel to the central curve. If $\varpi e^{\imath p t}$ be the total current, and $(\mathrm{L}, \mathrm{R})$ the effective self-induction and resistance per unit length of wire,

$$
(\mathrm{L} \iota p+\mathrm{R}) \varpi=\mathrm{E} . \quad \cdot \quad \cdot \quad \cdot
$$

Now at this point, the problem becomes identical in process with that of the straight wire, provided that the appropriate functions $\mathrm{A}_{0}(k \rho)$ and $\mathrm{B}_{0}(\iota h \rho)$ are used for the wire and dielectric respectively, in place of $J_{0}(k \rho)$ and $K_{0}(\iota h \rho)$ for the straight wire. Quoting therefore the result in the latter case, as developed in a former paper ${ }^{*}$, namely, if $\boldsymbol{r}$ be the radius of the wire,

$$
\frac{\mathrm{E}}{\sigma}=\frac{2 \mu \iota p}{k r}\left\{\frac{J_{0}(k r)}{J_{0}^{\prime}(k r)}-\frac{k}{\mu h \iota} \frac{\mathrm{K}_{0}(\iota h r)}{\overline{\mathrm{K}}_{0}^{\prime}(\iota h r)}\right\}, .
$$

we find for the helix

$$
\frac{\mathrm{E}}{\sigma}=\frac{2 \mu \iota p}{k r}\left\{\frac{\mathrm{A}_{0}(k r)}{\mathrm{A}_{0}^{\prime}(k r)}-\frac{k}{\mu h \iota} \frac{\mathrm{B}_{0}(\iota h r)}{\mathrm{B}_{0}^{\prime}(\iota h r)}\right\} \ldots
$$

* Phil. Mag. Feb. 1909, p. 259. The result requires a factor $-1 / a$. 
Let

$$
16 \delta=\cos ^{2} \alpha\left(5 \sin ^{2} \alpha-1\right), . . .
$$

Then

$$
\begin{aligned}
& A_{0}(l r r)=\left(1+\frac{\delta r^{2}}{a^{2}}\right) J_{0}(k r) \\
& A_{0}^{\prime}(k r)=\left(1+\begin{array}{c}
\delta r^{2} \\
a^{2}
\end{array}\right) J_{0}^{\prime}(k r)+\frac{2 \delta r}{k a^{2}} \mathrm{~J}_{0}(k r),
\end{aligned}
$$

and to order $1 / k^{2} a^{2}$

$$
\frac{A_{0}(k r)}{A_{0}^{\prime}(k r)}=\frac{J_{0}(k r)}{J_{0}{ }^{\prime}(k r)}\left\{1-\frac{2 \delta r}{k a^{2}} \frac{J_{0}(k r)}{J_{0}{ }^{\prime}(k r)}\right\} .
$$

Finally, if

$$
\begin{gathered}
\frac{\mathrm{J}_{0}(k r)}{k r J_{0}^{\prime}(k r)}=\mathrm{P}+\iota \mathrm{Q}, \quad \frac{\mathrm{K}_{0}(\iota h r)}{\iota h r \mathrm{~K}_{0}^{\prime}(\iota h r)}=\mathrm{P}^{\prime}+\iota \mathrm{Q}^{\prime}, . \\
\frac{\mathrm{E}}{\sigma}=2 \mu \iota p(\mathrm{P}+\iota \mathrm{Q})\left\{1-\frac{2 \delta r^{2}}{a^{2}}(\mathrm{P}+\iota \mathrm{Q})\right\} \\
-2 \imath p\left(\mathrm{P}^{\prime}+\iota \mathrm{Q}^{\prime}\right)\left\{1-\frac{2 \delta r^{2}}{a^{2}}\left(\mathrm{P}^{\prime}+\iota \mathrm{Q}^{\prime}\right)\right\} .
\end{gathered}
$$

In determining the effect of the concentration of current upon the inductance and resistance, which in the former case is all that is sought, only the terms involving $\mathbf{P}$ and $\mathbf{Q}$ (and thus the resistivity) are needed. The effective resistance is altered from its steady current value of $\sigma / \pi r^{2}$ to the real part of $\mathrm{E} / \sigma$ or

$$
\mathrm{R}=-2 \mu p \mathrm{Q}+\frac{\mu p r^{2}}{4 a^{2}} \cos ^{2} \alpha\left(1-5 \sin ^{2} \alpha\right) \mathrm{PQ}, .
$$

and the self-induction is

$$
\mathrm{L}=2 \mu \mathrm{P}-\frac{\mu r^{2}}{4 a^{2}} \cos ^{2} \alpha\left(1-5 \sin ^{2} \alpha\right)\left(\mathrm{P}^{2}-\mathrm{Q}^{2}\right) .
$$

The values of $P$ and $Q$ are well known. With Lord Kelvin's definition of the functions ber $x$, bei $x$, where

$$
\begin{aligned}
& x=\iota-3 r=r(4 \pi \mu p / \sigma)^{\frac{2}{2}}, \\
& \mathrm{~J}_{0}(k r)=\text { ber } x+\iota \text { bei } x, \quad . \quad . \quad .
\end{aligned}
$$

and on reduction,

$$
\left.\begin{array}{rl}
-x \mathrm{Q} & =\left(\text { ber } x \text { bei' } x-\text { bei } x \operatorname{ber}^{\prime} x\right) /\left\{\left(\operatorname{ber}^{\prime} x\right)^{2}+\left(\operatorname{bei}^{\prime} x\right)^{2}\right\} \\
x \mathrm{P} & =\left(\text { ber } x \text { ber' } x+\text { bei } x \operatorname{bei}^{\prime} x\right) /\left\{\left(\operatorname{ber}^{\prime} x\right)^{2}+\left(\operatorname{bei}^{\prime} x\right)^{2}\right\}
\end{array}\right\}
$$


The values of these functions have been tabulated, yet as Russell has shown *, the tables need revision. But it is preferable, in place of a laborious tabulation, to use the asymptotic formulæ for these functions, which Russell develops in the same paper. We may distinguish two important cases, and it is convenient to recapitulate, at this stage, the meanings of the various symbols. The oscillation is assumed to be sine-shaped, and of frequency $p / 2 \pi, \mu$ and $\sigma$ are the permeability and resistivity of the wire. The wire has a radius $r$ of section, and is wound on a cylinder of radius $a$, such that $r / a$ is small. The angle of the helix is $a$. Let $n$ be the number of turns of the helix in a length $z$ parallel to its axis. Since, in a helix, $z=a \theta \tan \alpha$, we have

and therefore

$$
n=\theta / 2 \pi=z / 2 \pi a \tan \alpha \text {, }
$$

$$
\alpha=\tan ^{-1}(z / 2 \pi n a) . \quad . \quad . \quad . \quad \text {. }
$$

Case of Small Frequency.

When $x=r(4 \pi \mu p / \sigma)^{\frac{2}{2}}$ is less than 2 , we may write

$$
\left.\begin{array}{l}
x \mathrm{P}=\frac{1}{4} x\left\{1-\frac{1}{24}\left(\frac{x}{2}\right)^{4}+\frac{13}{4320}\left(\frac{x}{2}\right)^{8}-\frac{647}{12.360 .56}\left(\frac{x}{2}\right)^{12}\right\} \\
x \mathrm{Q}=\frac{2}{x}\left\{1+\frac{1}{12}\left(\frac{x}{2}\right)^{4}-18\left(\frac{x}{2}\right)^{8}+\frac{11}{12.28 .30}\left(\frac{x}{2}\right)^{12}\right\}
\end{array}\right\},
$$

and therefore

$$
\begin{aligned}
\mathrm{PQ}= & -\frac{1}{2 x^{2}}\left\{1+\frac{1}{24}\left(\frac{x}{2}\right)^{4}-\frac{13}{2160}\left(\frac{x}{2}\right)^{8}\right\} \\
\mathrm{P}^{2}-\mathrm{Q}^{2}= & \frac{1}{16}\left\{1-\frac{1}{12}\left(\frac{x}{2}\right)^{4}+\frac{67}{8640}\left(\begin{array}{l}
x \\
2
\end{array}\right)^{8}\right\} \\
& -\frac{4}{x^{2}}\left\{1+\frac{1}{6}\left(\frac{x}{2}\right)^{4}-\frac{1}{240}\left(\frac{x}{2}\right)^{8}\right\},
\end{aligned}
$$

no higher order being necessary in the last two, as they will be multiplied by $r^{2} / a^{2}$.

The resistance becomes

$$
\begin{gathered}
\mathrm{R}=\frac{\sigma}{\pi r^{2}}\left\{1+\frac{1}{12}\left(\frac{\pi \mu p r^{2}}{\sigma}\right)-\frac{1}{180}\left(\frac{\pi \mu p r^{2}}{\sigma}\right)+\frac{11}{12.28 .30}\left(\frac{\pi \mu p r^{2}}{\sigma}\right)^{6}\right\} \\
-\begin{array}{c}
\sigma \\
32 \pi a^{2}
\end{array} \cos ^{2} \alpha\left(1-5 \sin ^{2} \alpha\right)\left\{1+\frac{1}{24}\left(\frac{\pi \mu p r^{2}}{\sigma}\right)^{2}-\frac{13}{2160}\left(\frac{\pi \mu p r^{2}}{\sigma}\right)^{4}\right\},(42) \\
\text { * Loc. cit.; }
\end{gathered}
$$


and the self-induction is changed by the concentration of current to an amount

$$
\begin{aligned}
\mathrm{L} & =\frac{1}{2} \mu\left\{1-\frac{1}{24}\left(\frac{\pi \mu p r^{2}}{\sigma}\right)^{2}+\frac{13}{4320}\left(\begin{array}{c}
\pi \mu p r^{2} \\
\sigma
\end{array}\right)^{4}-\frac{647}{122.360 .56}\left(\frac{\pi \mu p r^{2}}{\sigma}\right)^{6}\right\} \\
& -\frac{\mu r^{2}}{64 a^{2}} \cos ^{2} \alpha\left(1-5 \sin ^{2} \alpha\right)\left\{1-\frac{1}{12}\left(\begin{array}{c}
\pi \mu p r^{2} \\
\sigma
\end{array}\right)^{2}+\frac{67}{8640}\left(\begin{array}{c}
\pi \mu p r^{2} \\
\sigma
\end{array}\right)^{4}\right\} \\
& +\frac{\sigma}{4 \pi \alpha^{2} p} \cos ^{2} \alpha\left(1-5 \sin ^{2} \alpha\right)\left\{1+\frac{1}{6}\left(\frac{\pi \mu p r^{2}}{\sigma}\right)^{2}-\frac{1}{240}\left(\frac{\pi \mu p r^{2}}{\sigma}\right)^{4}\right\} \cdot(43)
\end{aligned}
$$

In these results it is not possible to proceed to the limit of zero frequency, for it has been assumed that $k^{2} a^{2}$ is large. Although, therefore, the expression for $\mathbf{R}$ does not become $\sigma / \pi r^{2}$ when $p$ is made zero, no error is involved on this account. Near $p=0$ another form of expression must be used.

The limitations of these results are determined by the considerations that $k r^{2}$ shall be less than 2 , and that $r^{3} / a^{3}$ and $\mathrm{ka}^{-4}$ shall be negligible. As an average practical case, we take a copper wire of radius 2 millimetres, and determine the requisite conditions for a three figure accuracy. In the first place, $r^{3} / a^{3}$ cannot be ignored, unless $a$ is greater than $12 r$ approximately. This condition is usually fulfilled. Even if it is not, the order of accuracy may extend to two figures for a much greater value of $r / a$, so that this limitation is not of great moment.

Secondly, $k r$ is less than 2 , so that in the present case, if $f$ be the frequency, and $\sigma=1696$ c.G.s. units, approximately

$$
\frac{1}{3} r f^{\frac{1}{2}}<2 \text { or } \quad r f^{\frac{1}{2}}<10,
$$

where $r$ is in centimetres. The upper limit in frequency is therefore about 2500 per second.

Thirdly. $(k a)^{-4}$ may be neglected if $k a>10$, so that, to determine the lower limit, there is an inequality

$$
a f^{\frac{1}{2}}>50 \text {, }
$$

or if $a=12 r$ in the most unfavourable case, $f$ is about 400 per second. The formulæ, therefore, range between frequencies of 400 and 2500 for a wire of 2 millimetres radius, wound on an appropriate cylinder. In general the conditions for a three figure accuracy are

$$
a>12 r, \quad a f^{\frac{1}{2}}>50, \quad r f^{\frac{1}{2}}<10, . . .
$$

the radii being in centimetres. 
Case of High Frequency.

We pass to the case of a higher frequency, whose lower limit is determined later. When

$$
\lambda=x 2^{\frac{1}{2}}=2 r(2 \pi \mu p / \sigma)^{\frac{1}{2}}
$$

is not less than 8 , a four figure accuracy may be obtained by the use of the formulæ*

$$
\left.\begin{array}{rl}
\lambda \mathrm{P} & =1-\frac{3}{4 \lambda^{2}}-\frac{3}{2 \lambda^{3}} \\
-\lambda \mathrm{Q} & =1+\frac{1}{\lambda}+\frac{3}{4 \lambda^{2}}
\end{array}\right\},
$$

a small but convenient change in the notation having been introduced. Therefore

and finally

$$
\begin{aligned}
& -\lambda^{2} \mathrm{PQ}=1+\frac{1}{\lambda}-\frac{9}{4 \lambda^{3}} \\
& \lambda^{2}\left(\mathrm{P}^{2}-\mathrm{Q}^{2}\right)=-\left(\frac{2}{\lambda^{2}}+\frac{4}{\lambda^{2}}+\frac{9}{4 \lambda^{3}}\right),
\end{aligned}
$$

$$
\begin{aligned}
\mathrm{R} & =\left(\frac{\mu p \sigma}{2 \pi r^{2}}\right)^{\frac{2}{2}}\left\{1+\left(\frac{\sigma}{8 \pi \mu p r^{2}}\right)^{\frac{1}{2}}+\frac{3}{4} \cdot \frac{\sigma}{8 \pi \mu p r^{2}}\right\} \\
& -\frac{\sigma}{32 \pi a^{2}} \cos ^{2} \alpha\left(1-5 \sin ^{2} \alpha\right)\left\{1+\left(\frac{\sigma}{8 \pi \mu p r^{2}}\right)^{\frac{2}{2}}-\frac{9}{4}\left(\frac{\sigma}{8 \pi \mu p r^{2}}\right)^{\frac{3}{2}}\right\} \\
\mathrm{L} & =\left(\frac{\sigma \mu}{2 \pi r^{2}}\right)^{\frac{1}{2}}\left\{1-\frac{3 \sigma}{48 \pi \mu r^{2}}-\frac{3}{2}\left(\frac{\sigma}{\delta \pi \mu p r^{2}}\right)^{\frac{3}{2}}\right\} \\
& +\frac{\sigma \cos ^{2} \alpha\left(1-5 \sin ^{2} \alpha\right)}{32 \pi p r \alpha^{2}}\left(\frac{\sigma}{2 \pi \mu p}\right)^{\frac{2}{2}}\left\{1+2\left(\frac{\sigma}{8 \pi \mu p r^{2}}\right)^{\frac{1}{2}}+\frac{9}{48 \pi p r^{2}}\right\}
\end{aligned}
$$

A three-figure accuracy in these results will be secured if the conditions

$$
a>12 r, \quad r f^{\frac{2}{2}}>70 .
$$

are satisfied, in the case of copper wires. No further condition as regards $\boldsymbol{a}$ is here needed, as it is satisfied by virtue of the others. In the limiting case of very high frequency

$$
\begin{gathered}
\mathbf{R}=\left(\frac{\mu p \sigma}{2 \pi r^{2}}\right)^{\frac{1}{2}}-\frac{\sigma}{32 \pi a^{2}} \cos ^{2} \alpha\left(1-5 \sin ^{2} \alpha\right) . . . . \\
\mathbf{L}=\left(\frac{\sigma \mu}{2 \pi p r^{2}}\right)^{\frac{1}{3}}+\frac{\sigma \cos ^{2} \alpha\left(1-5 \sin ^{2} \alpha\right)}{32 \pi p r a^{2}}\left(\frac{\sigma}{2 \pi \mu p}\right)^{\frac{3}{2}}-\frac{3 \sigma}{32 \pi \mu p r^{2}}\left(\begin{array}{c}
\sigma \mu \\
2 \pi p r^{2}
\end{array}\right)^{\frac{1}{2}},(50) \\
\text { Russell, l. c. p. 532. }
\end{gathered}
$$


and the change in resistance due to twisting tends to become independent of both the frequencr and the radius of the wire, whereas the change in self-induction tends to vanish.

For the intermediate onse, in which $r f^{\frac{1}{2}}$ lies between the limits of about 10 and 70 , the formulæ $(36,37)$ must be used, provided that $a$ satisfies the proper condition. These formulæ require the functions $\mathrm{P}$ and $\mathrm{Q}$ when $x$ lies between 2 and 7 .

In a paper recently presented to the Physical Society*, Mr. H. G. Savidge has given tables of these functions suitable for the case in question, together with graphs of the functions $-x \mathrm{Q}, x \mathrm{P}$ of (39). The formulæ (36), (37) are therefore rendered sufficient for practical use.

\section{Note on a Gravitational Problem. By C. V. Burton, D.Se.†}

1. WITHOUT actual loss of generality, the question V. here considered may be stated as follows :- If the action of gravity were intermittent in character, the mutual attraction of any two bodies fluctuating between zero and twice its mean value, how long could the period of the fluctuation be without giring rise to observable periodic efferts? At a first glance we might be tempted to reply that the period could only be a very small fraction of a second; but though this answer would be justified if the earth were perfectly rigid, the yielding of the earth to pressure and to shearing-stress completely changes the aspect of the problem, and the results obtained are so different from what might have been hastily assumed, that a brief indication of them may possess some interest.

2. Let us suppose that the gravitation-constant G represents merely the mean value of $\mathrm{G}\left(1+\mathrm{A} e^{i p t}\right)$, where $\mathrm{A}$ is a numerical constant; so that, at any time $t$, the attraction between masses $m, m^{\prime}$ at a distance $r$ apart is

$$
\mathrm{G}\left(1+\mathrm{A} e^{i p t}\right) m m^{\prime} / r^{2} \text {. }
$$

For the sake of simplicity the earth will now be regarded as spherical and sensibly homogeneous; and accordingly the intensity of the gravitative field at any point of the surface at time $t$ will be

$$
g\left(1+A e^{i p t}\right) ; \quad \cdot \quad \cdot \quad \cdot \quad \cdot
$$

* Suprà, p. 49.

$\uparrow$ Communicated by the Physical Society : read Norember 26, 1909. 